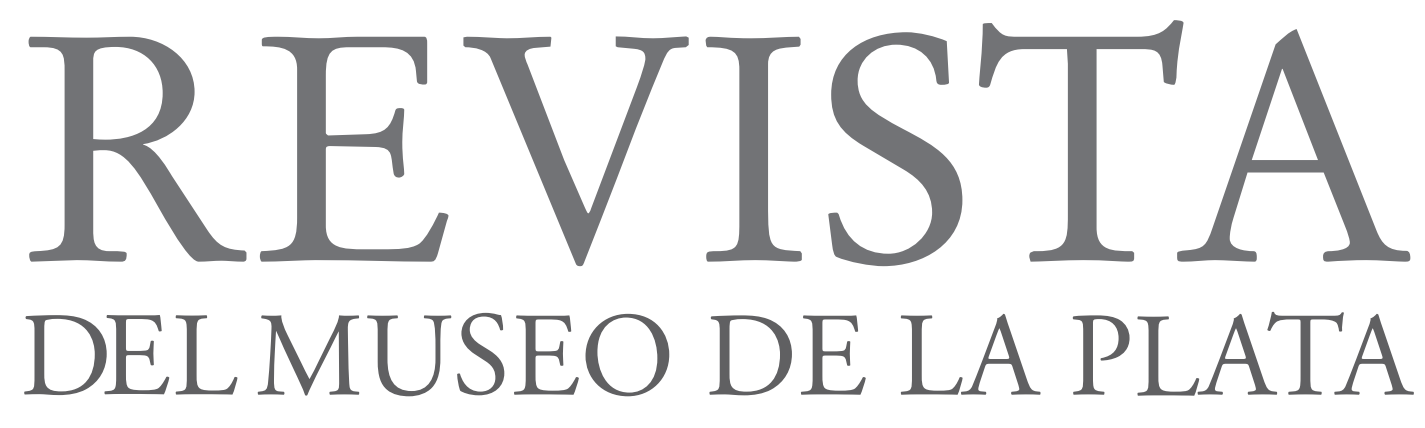

UNLP | Facultad de Ciencias Naturales y Museo

\author{
2018 \\ VOL. 3, NÚM. 1, SUPLEMENTO RESÚMENES
}

TALLER IV: APORTES Y DISCUSIONES EN ZOOARQUEOLOGÍA (19 de octubre, La Plata)

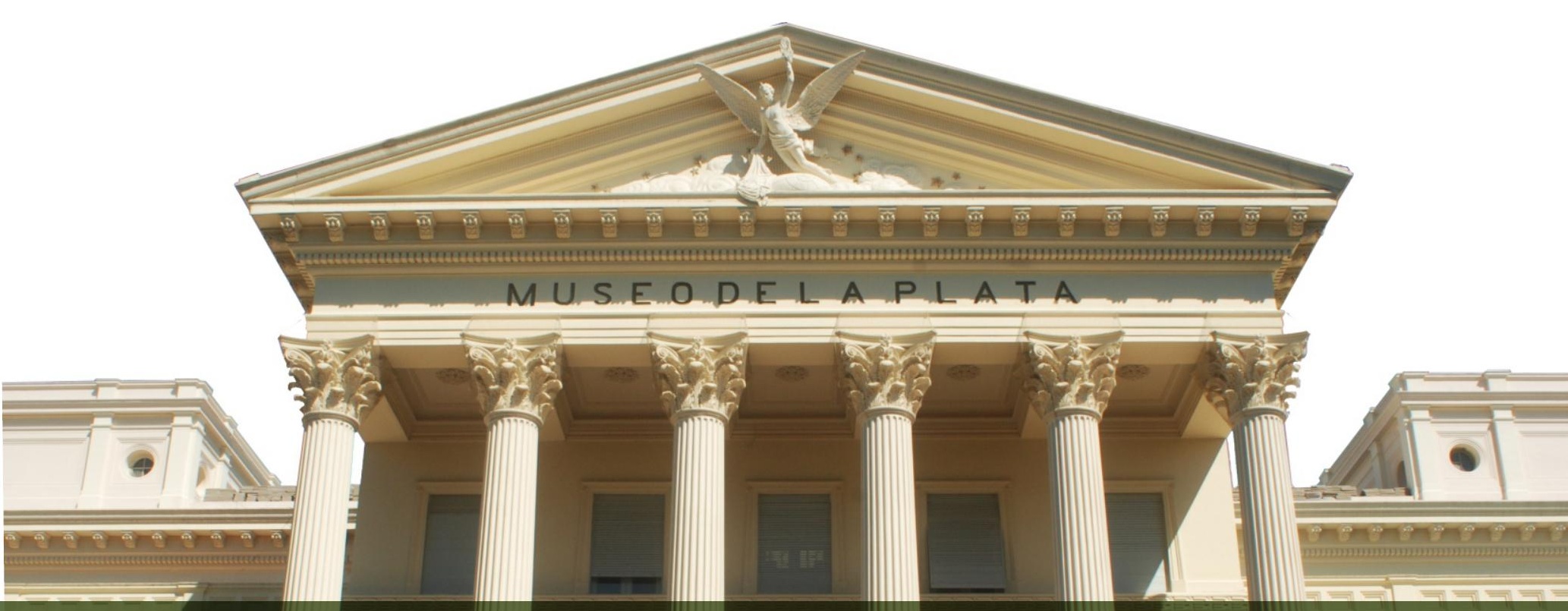




\author{
CICLO DE TALLERES \\ DE LA DIVISIÓN ARQUEOLOGÍA \\ Museo de La Plata, Facultad de Ciencias naturales y Museo
}

\title{
TALLER IV: APORTES Y DISCUSIONES EN ZOOARQUEOLOGÍA
}

\section{LIBRO DE RESÚMENES}

19 de OCtubre de 2018, Museo de La Plata

\section{ORGANIZADO POR}

División Arqueología, Museo de la Plata

Facultad de Ciencias Naturales y Museo, Universidad Nacional de La Plata

Lic. Eloisa García Añino

Lic. Emiliano Mange

Dra. Laura Marchionni

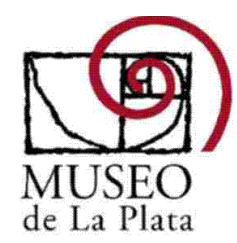




\title{
Animales y ritualidad en el mundo inka. Un caso de estudio en el sitio arqueológico El Shincal de Quimivil (Londres, Catamarca)
}

\author{
M. V. Valderrama ${ }^{1}$, M. A. Giovannetti ${ }^{1,2}$ y M. A. Loperfido ${ }^{1}$ \\ ${ }^{1}$ División Arqueología, Facultad de Ciencias Naturales y Museo, Universidad Nacional de La Plata \\ mvalderrama27@gmail.com; marcogiovannetti@gmail.com; marcoloperfido1@hotmail.com \\ ${ }^{2}$ CONICET
}

El presente trabajo tiene como objetivo indagar las posibles relaciones que se forjaron entre personas humanas y no humanas (Hallowell 1960) en un contexto arqueológico particular: Recinto 62, Complejo 17 del sitio El Shincal de Quimivil. El mismo pretende discutir una posible interpretación de los restos arqueofaunísticos, relacionándolos con prácticas de uso y consumo en un contexto ritual.

El Shincal de Quimivil se emplaza en el sector occidental de la Provincia de Catamarca, departamento de Londres, sobre el extremo noroeste de un amplio cono aluvial, delimitado por el curso de los ríos Quimivil y Hondo (Giovannetti 2009).

Las múltiples investigaciones realizadas en el sitio, permiten interpretarlo como un centro de importancia política y administrativa en la estructura del Tawantinsuyu (Raffino 2004; Giovannetti 2009). Los estudios realizados recientemente han permitido, además, explorar un posible rol ritual donde se celebraban las importantes fiestas del Calendario Oficial. En las mismas se compartían abundantes cantidades de comidas y bebidas entre diversas comunidades (Giovannetti 2009, Giovannetti et al. 2012). Aquí, los restos faunísticos juegan un importante papel para la interpretación de las prácticas de comensalismo (Bray 2012).

Los resultados zooarqueológicos obtenidos contribuyen a la discusión acerca del uso y consumo faunísticos en el pasado inkaico, específicamente en aquellos eventos que exceden la cotidianeidad. Su articulación con el análisis de otros elementos (cerámica, rasgos arquitectónicos y restos arqueobotánicos), junto a su disposición espacial, ha permitido destacar el carácter ritual del contexto.

Palabras claves: Zooarqueología, Ritualidad, Inka, Festividad, Comensalismo 


\title{
Identificación específica de Rheidae en Boyo Paso 2 ca. 1500 - 750 AP (Sierras de Córdoba, Argentina): implicancias económicas y paleoambientales
}

\author{
M. Medina ${ }^{1,2}$, M. Picasso ${ }^{2,3}$, N. Ávila ${ }^{4}$ y M. Campos ${ }^{4}$ \\ ${ }^{1}$ División Arqueología, Facultad de Ciencias Naturales y Museo, Universidad Nacional de La Plata, Argentina. \\ paleomedina@gmail.com \\ ${ }^{2}$ CONICET \\ ${ }^{3}$ División Paleontología de Vertebrados, Facultad de Ciencias Naturales y Museo, Universidad Nacional de La Plata, Argentina. \\ mpicasso@fcnym.unlp.edu.ar \\ ${ }^{4}$ Facultad de Filosofía y Letras, Universidad de Buenos Aires, Argentina. nancyamasuno@hotmail.com; mailinrcampos@gmail.com
}

Se presentan los resultados del estudio realizado sobre los restos de Rheidae recuperados en Boyo Paso 2 (Sierras de Córdoba, Argentina), un sitio a cielo abierto con ocupaciones asignadas al Periodo Prehispánico Tardío (1500-360 AP). El objetivo fue identificar las especies presentes y discutir la diversidad taxonómica de la subsistencia tardía, así como las condiciones paleoambientales con la que grupos de economía mixta interactuaron a fines del Holoceno. Un fragmento de tarsometatarso distal y otro proximal provenientes de pisos arqueológicos son descriptos y comparados con especímenes actuales de ñandú común (Rhea americana) y ñandú petiso ( $R$. pennata). La similitud morfológica entre las especies de Rheidae dificultó la identificación precisa de los especímenes arqueológicos. La determinación taxonómica de cáscaras de huevo se realizó a partir del estudio de la densidad de los poros, asignando los especímenes a una u otra especie de acuerdo a intervalos definidos para muestras de $R$. americana y $R$. pennata. Una pequeña fracción del conjunto fue identificada como Rhea $c f . R$. americana y Rhea cf. $R$. pennata, siendo esta última no citada en la literatura biogeográfica de las Sierras de Córdoba. Los resultados indicaron que la adopción de cultivos ca. 1.500 años AP fue acompañada por la explotación y consumo de una mayor diversidad de recursos que lo tradicionalmente observado, incluyendo especies nunca antes consideradas como $R$. pennata. La identificación de este taxón fuera de su área de distribución actual indica que el paisaje de fines del Holoceno presentaba una mayor biodiversidad para la cual el ambiente moderno no es un buen análogo. Lograr determinaciones más precisas, de esta manera, se convierte en uno de los pilares fundamentales para estimar la riqueza taxonómica de la subsistencia humana e interpretar la biodiversidad de los ambientes serranos de fines del Holoceno.

Palabras claves: Sierras de Córdoba, Periodo prehispánico tardio, Rheidae, Identificación taxonómica, Subsistencia, Paleoambiente 


\title{
Estado actual y perspectivas de las investigaciones zooarqueológicas en el Chaco argentino
}

\author{
M. M. Zarza ${ }^{1}$, L. M. Del Papa ${ }^{1,2}$, G. N. Lamenza ${ }^{1,2}$ \\ ${ }^{1}$ Facultad de Ciencias Naturales y Museo. Universidad Nacional de La Plata, Argentina. \\ macarena_zarza@hotmail.com; loescharquero@yahoo.com.ar; guillermolamenza@gmail.com \\ ${ }^{2}$ CONICET
}

Las últimas investigaciones arqueológicas desarrolladas en el Gran Chaco argentino ponen en valor a la región como partícipe activo en los procesos de desarrollo cultural sudamericano. El modelo general propone que, desde el Holoceno tardío, habitaron grupos cazadoresrecolectores-pescadores con movilidad estacional, fluctuante según niveles diferenciales de inundación, con explotación de recursos previsibles y vinculados a un sistema de subsistencia ribereño. Sin embargo, aún resta esclarecer aspectos que no han sido abordados previamente tales como la resolución temporal del registro; la correlación entre los componentes culturales con la explotación diferencial de recursos; el análisis comparativo de las distribuciones pasadas y actuales; las posibles variaciones morfológicas de los taxones identificados a lo largo de la secuencia; entre otros. En esta oportunidad se presenta el estado actual del conocimiento y las perspectivas de las investigaciones zooarqueológicas en el Chaco argentino. Asimismo, a modo de ejemplo, se dan a conocer algunos materiales que permitirán plantear interrogantes y discutir sobre los procesos tafonómicos, las técnicas de manufactura y la posibilidad de utilización de soportes óseos con fines decorativos en vinculación con el campo de las representaciones simbólicas.

Palabras clave: Tierras bajas sudamericanas, Holoceno tardio, Cazadores recolectores pescadores 


\title{
Del registro ictioarqueológico a las prácticas alimentarias: el caso de Los Tres Cerros 1 (Delta Superior del Paraná, Entre Ríos)
}

\author{
L. Bastourre ${ }^{1}$ \\ ${ }^{1}$ División Arqueología, Facultad de Ciencias Naturales y Museo, Universidad Nacional de La Plata, Argentina. \\ laurabastourre@yahoo.com.ar
}

El sitio Los Tres Cerros 1 es un montículo antrópico que fue ocupado entre 1030 y 560 años AP por grupos con una subsistencia basada en la caza, la pesca, la recolección y la horticultura a pequeña escala. El objetivo de este trabajo es abordar el componente ictiofaunístico de la alimentación, y, en particular, analizar las trayectorias que atravesaron estos recursos desde la obtención de los peces hasta su descarte, incluyendo las prácticas involucradas en su procesamiento. Indagar esta temática implicó también comprender el conjunto de los procesos naturales que afectaron los restos una vez depositados. Con estos fines, se analizó la abundancia taxonómica y los perfiles anatómicos en conjunto con variables como meteorización, fragmentación, densidad ósea e índices morfológicos. Asimismo, se puso énfasis en el estudio de la distribución de las huellas de procesamiento y las evidencias de termoalteración. Los análisis muestran el aprovechamiento de numerosas especies de Characiformes (mayormente Hoplias sp. y Prochilodus lineatus y, en menor medida, Leporinus obtusidens, Salminus brasiliensis, Piaractus mesopotamicus, entre otras), Siluriformes principalmente bagres (como Pimelodus maculatus, P. albicans y Rhamdia quelen) y armado común (Pterodoras granulosus), así como varias especies de calíctidos, auqueniptéridos y loricáridos- y Perciformes (Cichlidae). Estos recursos fueron obtenidos mediante variadas técnicas de captura, enfocadas principalmente en los sectores lénticos del paisaje fluvial. Posteriormente, fueron procesados a través de actividades como la decapitación, el fileteo y el trozamiento, y preparados mediante distintas técnicas de cocción (hervido, asado) y, probablemente, de conservación a largo plazo. El descarte de los restos producidos a partir de estas actividades involucró el uso de sectores específicos del espacio (i.e. basureros). Se registraron diferentes tendencias para las variables analizadas entre los conjuntos ictiológicos recuperados en distintos sectores del sitio. Algunas de estas tendencias fueron atribuidas a procesos tafonómicos naturales, mientras que otras fueron interpretadas en términos de diferencias en las secuencias de procesamiento, consumo y descarte de los distintos peces.

Palabras clave: Ictioarqueología, Delta Superior del Paraná, Procesos de formación, Cerritos, Holoceno tardio 


\title{
Estudios arqueofaunísticos en sitios del litoral del Río de la Plata
}

\author{
F. Day Pilaría ${ }^{1}$ \\ ${ }^{1}$ Laboratorio de Análisis Cerámico, Facultad de Ciencias Naturales y Museo, Universidad Nacional de La Plata. \\ fernandaday@yahoo.com.ar
}

El objetivo de esta exposición es presentar una síntesis de los análisis realizados con los materiales arqueozoológicos recuperados en los sitios costeros de los actuales partidos de Magdalena y Punta Indio. Los estudios se centraron en comprender cuáles fueron las estrategias organizativas desarrolladas por las sociedades cazadoras, recolectoras y pescadoras durante distintos momentos de ocupación del Holoceno tardío, cómo gestionaron los recursos faunísticos y cómo fueron las prácticas socioeconómicas que implementaron. Se identificaron una importante variedad de taxones (e.g. peces, reptiles, anuros y ofidios, aves, distintas especies de mamíferos de diversos tamaños). Asimismo, se visualizaron diferentes tendencias en la explotación de los recursos animales según el momento de ocupación de los sitios, como así también en la gestión, estrategias y prácticas involucradas. Una particularidad de los conjuntos arqueozoológicos con antigüedades próximas a los 1000 años AP, es que presentan proporciones considerablemente menores de restos de peces. Este es un aspecto que se comienza a explorar en relación a los posibles cambios paleoambientales que pudieran haber ocurrido en el área de estudio.

La información arqueofaunística generada se articuló e integró con otras líneas de evidencia como la cerámica, material lítico, análisis de microrrestos y de ácidos grasos, y con distintas fuentes etnográficas y etnohistóricas.

Se presentarán una serie de instrumentos óseos recuperados en el sitio Las Marías (Magdalena), y otros especímenes que evidencian procesos tafonómicos particulares (e.g. pérdida de tejido cortical debido al rodamiento por una intensa acción fluvial, coloración brillosa por la incorporación de minerales que se encuentran en el agua del río).

Otra característica particular de los conjuntos analizados es la baja proporción de modificaciones de origen antrópico (e.g. fracturas frescas, marcas de corte, de percusión, negativos de impacto y lascados). En este sentido, se encuentran en desarrollo una serie de estudios experimentales que permitirán interpretar las prácticas de procesamiento y cocción de especies animales identificadas en el registro arqueofaunístico.

Asimismo, en el ámbito de la zooarqueología histórica, se analizaron materiales provenientes de contextos rurales y urbanos del siglo XIX. Particularmente, en el sitio El Santuario I (Magdalena) se identificó una representación diferencial de partes esqueletarias de ganado vacuno y ovino, que se interpreta, a través de la integración con fuentes documentales, como un posible manejo de estas especies en espacios diferenciados de la estancia.

Palabras clave: Recursos faunísticos, Estrategias socioeconómicas, Río de la Plata, Holoceno tardio 


\title{
Tafonomía actualística de guanacos en el Sistema Serrano de Ventania, provincia de Buenos Aires
}

\author{
N. S. Morales ${ }^{1,2}$, L. Catella ${ }^{1,2,3}$ y G. Barrientos ${ }^{1,2,3}$ \\ ${ }^{1}$ División Arqueología, Facultad de Ciencias Naturales y Museo, Universidad Nacional de La Plata, Argentina. \\ ${ }^{2}$ CEAR, Facultad de Humanidades y Artes, Universidad Nacional de Rosario, Argentina. \\ moralesnatalia@fcnym.unlp.edu.ar; catellaluciana@hotmail.com; gustavbarrie@yahoo.com.ar \\ ${ }^{3}$ CONICET
}

La observación y análisis de los procesos de modificación de los cuerpos de animales muertos bajo diferentes circunstancias y en distintos contextos ambientales (i.e. tafonomía actualística en modo naturalista), ha constituido un tema recurrente de investigación durante los últimos 40 años, tanto a nivel mundial como local. Estos estudios han permitido discutir diferentes problemas de naturaleza paleoecológica y zooarqueológica. En este marco, el objetivo de este trabajo es presentar los resultados preliminares de una investigación, iniciada en 2016, orientada al estudio de los procesos tafonómicos actuantes sobre osamentas de guanaco depositadas en diferentes sectores del Parque Provincial Ernesto Tornquist (PPET), localizado en el Sistema Serrano de Ventania, en el sur de la provincia de Buenos Aires. Los propósitos de la investigación son: a) contribuir a ampliar la casuística referida a estudios tafonómicos longitudinales y transversales sobre guanacos, en un ambiente más cálido y húmedo que los hasta ahora evaluados en Fuego-Patagonia; b) reunir información relevante que pueda potencialmente perfeccionar las inferencias arqueológicas efectuadas sobre conjuntos óseos de guanaco en el área de Ventania y zonas adyacentes de la Región Pampeana. La población de guanacos del PPET es pequeña, calculándose actualmente un tamaño de alrededor de 50 individuos (densidad aproximada de $0,75 \mathrm{ind} / \mathrm{km} 2)$. Durante los trabajos de campo $(\mathrm{n}=9)$, se localizaron seis osamentas en diferente estado de desarticulación, la mayoría de las cuales fueron visitadas en más de una oportunidad. En cada caso, se procedió a efectuar la georreferenciación, el registro fotográfico y cartográfico y el relevamiento de variables cualicuantitativas de interés. Durante la presentación, se discutirán las técnicas de análisis espacial y estadístico aplicadas para evaluar la dispersión y supervivencia de partes esqueletarias, así como las similitudes y diferencias, en términos tanatológicos y tafonómicos, respecto de los resultados obtenidos por otros estudios similares en diferentes regiones del Cono Sur.

Palabras clave: Tafonomía, Guanacos, Región Pampeana, Análisis espacial, SIG 


\title{
El registro faunístico y la funcionalidad de las estructuras de piedra del sitio Recintos de Aguada (piedemonte de Somuncurá, Río Negro)
}

\author{
M. Di Lorenzo ${ }^{1}$, E. Mange ${ }^{1,2}$ y L. Prates ${ }^{1,2}$ \\ ${ }^{1}$ División Arqueología, Facultad de Ciencias Naturales y Museo, Universidad Nacional de La Plata \\ maiten.dilorenzo@gmail.com; lprates@fcnym.unlp.edu.ar \\ ${ }^{2} \mathrm{CONICET}$
}

El sitio Recintos de Aguada se encuentra ubicado en cercanías de la localidad de Aguada Cecilio (departamento de Valcheta, provincia de Río Negro), en un pequeño remanente de meseta basáltica, en cercanías de un manantial. Estructuras similares se han registrado en otros sectores de la Patagonia, y se les han propuesto dos tipos de funciones principales: a) parapetos de caza de guanaco (Lama guanicoe) en sectores altos (Gradin 1971, Goñi et al. 2011, Cassiodoro et al. 2012) y, b) bases de toldos habitacionales (Gradin 1976, García \& Pérez de Micou 1980, Miotti et al. 2016). Estas estructuras habrían sido conservadas y reutilizadas en forma estacional a través de los años, desde casi 2000 años AP hasta tiempos históricos (Videla 2003, Boschin \& del Castillo Bernal 2005, Miotti et al. 2016). El objetivo de este trabajo es evaluar estas dos hipótesis generales para el sitio Recintos de Aguada a través del estudio del conjunto faunístico.

El registro arqueológico del sitio incluye 17 estructuras de piedra (recintos circulares o semicirculares, alineaciones de piedra y montículos) y numerosos artefactos (principalmente líticos y restos faunísticos, y escasos tiestos cerámicos), distribuidos en el interior y en cercanías de ellas. En el interior de uno de los recintos circulares grandes se excavaron dos cuadrículas de $1 \mathrm{~m}^{2}$ en las cuales se recuperaron en posición superficial y estratigráfica numerosos artefactos líticos y faunísticos, y escasos restos vegetales y cerámicos. A partir de una muestra de carbón se obtuvo un fechado de $1.740 \pm 36$ años AP. Los resultados muestran escasa diversidad taxonómica e indican que además de guanacos, se explotaron eufractinos (Chaetophractus villosus y Zaedyus pichiy) y en menor medida carnívoros (principalmente Lycalopex sp.). El análisis tafonómico muestra que el conjunto se encuentra altamente fragmentado y con incidencia importante de termoalteración. Los resultados obtenidos en este trabajo son compatibles con el uso de las estructuras circulares como espacios de habitación pero con una marcada asociación con actividades de caza, posiblemente compatibles con campamentos operativos (sensu Binford 1980).

Palabras clave: Restos faunísticos, Estructuras de piedra, Funcionalidad, Río Negro 


\title{
Aproximaciones al registro zooarqueológico en la costa norte de Santa Cruz
}

\author{
H. Hammond ${ }^{1,2}$, P. Ambrústolo ${ }^{1,2}$ y L. Zilio ${ }^{1,2}$ \\ ${ }^{1}$ División Arqueología, Facultad de Ciencias Naturales y Museo, Universidad Nacional de La Plata, Argentina. \\ heidihammond@gmail.com.ar; pambrustolo@hotmail.com; leandrozilio@yahoo.com.ar \\ ${ }^{2}$ CONICET
}

En la costa norte de la provincia de Santa Cruz las investigaciones zooarqueológicas se enfocaron especialmente en las evidencias recuperadas en dos tipos de sitios principales: concheros y sitios en abrigos rocosos (aleros y cuevas). A partir del año 2010 se desarrolla un programa de estudios arqueomalacológicos sistemáticos de restos de moluscos que conforman los concheros. Estas investigaciones se han enfocado en el reconocimiento de las especies presentes, análisis tafonómicos de los exoesqueletos de moluscos; algo novedoso en la arqueología de Patagonia, así como el desarrollo de estudios actualísticos. Hasta el momento, los resultados de las investigaciones arqueomalacológicas han permitido discutir cuestiones relacionadas con la subsistencia de las poblaciones cazadores recolectoras que habitaron el área, los procesos de formación de sitio, las formas de obtención de los moluscos, un posible proceso de intensificación en el uso del recurso en el Holoceno tardío, entre otras. En cuanto a la fauna vertebrada en los concheros predominan los animales marinos (pinnípedos y aves marinas).

Los estudios zooarqueológicos de restos procedentes de abrigos rocosos del área permitieron identificar, a pesar de su relativa cercanía al litoral atlántico, conjuntos caracterizados principalmente por la presencia de recursos faunísticos terrestres, tales como el guanaco y bajas frecuencias de recursos marinos. Estos estudios han aportado a una comprensión más amplia acerca del uso del espacio y de los recursos faunísticos por los grupos humanos que habitaron en el pasado en la costa y el interior del territorio.

Por último, recientemente se ha generado la ecología isotópica del área. Se obtuvieron valores de isótopos estables $(\delta 13 \mathrm{C}$ y $\delta 15 \mathrm{~N})$ de huesos de fauna marina y terrestre utilizados como alimento potencial por los cazadores recolectores. Los resultados permitieron contribuir a las interpretaciones dietéticas de las poblaciones humanas que habitaron en el área a partir de la comparación entre los valores isotópicos faunísticos y los de muestras humanas.

Palabras clave: Concheros, Abrigos rocosos, Arqueomalacología, Vertebrados, Ecología isotópica 


\title{
Nuevos datos sobre las estrategias de aprovechamiento de los guanacos durante las ocupaciones tempranas del sitio AEP-1 de Piedra Museo (Macizo del Deseado, Santa Cruz)
}

\author{
L. Marchionni ${ }^{1,3}$, M. Vázquez ${ }^{2}$ y L. Miotti ${ }^{1,3}$ \\ ${ }^{1}$ División Arqueología, Facultad de Ciencias Naturales y Museo, Universidad Nacional de La Plata, Argentina. \\ lau_marchionni@yahoo.com.ar; laura.miotti2@gmail.com \\ ${ }^{\overline{2}}$ CADIC-CONICET vazquezmartin68@gmail.com \\ ${ }^{3}$ CONICET
}

El sitio AEP-1 de Piedra Museo corresponde a un alero rocoso a orillas de un paleolago ubicado en el noreste del Macizo del Deseado (Santa Cruz). Las investigaciones desarrolladas allí desde 1990 han contribuido con valiosa información acerca de las formas de vida de las sociedades cazadoras- recolectoras que habitaron la Patagonia desde los inicios del poblamiento en la transición Pleistoceno/Holoceno. Los trabajos realizados permitieron identificar dos componentes arqueológicos: el inferior y más antiguo, integrado por los conjuntos de la capa 6 (ca. 12.800 - 11.000 años AP) y la capa 4/5 (ca. 10.500 - 9200 años AP) y el superior, correspondiente al conjunto de la capa 2, referido al Holoceno medio ( $c a .7700$ - 7400 años AP). En este trabajo se presentan los resultados que estamos obteniendo a partir del análisis, a distintos aumentos, de las modificaciones de las superficies óseas presentes en los especímenes de Lama guanicoe (guanaco) de la capa 4/5. Estos nuevos estudios, tienen por objetivo identificar patrones de faenamiento y consumo, y por lo tanto, hay puesto un énfasis especial en aquellas modificaciones de origen antrópico. En este conjunto la abundancia relativa del guanaco es alta y en base a la representación y completitud anatómica, la distribución de los materiales en pilas, se propuso que el mismo sería resultado de actividades relacionadas con el procesamiento primario de presas cazadas en las inmediaciones del paleolago. A partir de los nuevos resultados re-evaluamos dicha hipótesis pudiendo ampliar las inferencias con la identificación de actividades no solo de procesamiento sino también de consumo de algunas partes de los animales que se faenaban en el lugar. De este modo, esperamos contribuir a la caracterización y discusión de las estrategias de explotación de los guanacos para momentos iniciales del poblamiento de la región.

Palabras clave: Indicadores de procesamiento, Camélidos, Cazadores- recolectores, Patagonia meridional 


\title{
Termoalteración como indicador de cocción para consumo. El caso del sitio Cueva Maripe (Santa Cruz): Implicancias y dificultades
}

\author{
E. García Añino ${ }^{1}$ \\ ${ }^{1}$ División Arqueología, Facultad de Ciencias Naturales y Museo, Universidad Nacional de La Plata, Argentina \\ eloisagarcia89@yahoo.com.ar
}

Existen diversos motivos por los cuales los huesos pueden haber sido expuestos a los efectos del calor, tanto intencionales como casuales, entre los que se incluyen la cocción, el descarte de los mismos en el fogón, su uso como combustible y las alteraciones postdepositacionales (Frank \& Paunero 2009; Langiano 2006; Marshall 1989; Merlo 2006; Wandsnider 1997). Cuando esto ocurre, los elementos óseos experimentan una serie de modificaciones graduales que se reflejan en una serie de atributos tanto macroscópicos como microscópicos, a partir de los cuales es posible aproximarse a las circunstancias en las que los elementos óseos han sido sometidos al calor. Sin embargo, en contextos habitacionales, con una recurrencia ocupacional elevada, el acercamiento a las circunstancias en que los especímenes fueron termoalterados puede resultar una tarea compleja. Este es el caso del sitio Cueva Maripe (Macizo del Deseado, provincia de Santa Cruz), de donde provienen los materiales analizados y presentados en este taller. Maripe es una cueva abierta, de gran envergadura, la cual se encuentra dividida en dos cámaras por un tabique rocoso (Cámara Norte y Cámara Sur). Los fechados radiocarbónicos sitúan a las ocupaciones humanas desde la transición PleistocenoHoloceno temprano ( $c$ a. 9.800 años C14 AP) hasta tiempos históricos.

Los análisis se centraron en aquellos especímenes que pudieron haber sido sometidos al calor como parte del proceso de cocción de los alimentos. La metodología utilizada para evaluar la termoalteración en los fragmentos óseos se basó en la propuesta de Cain (2005) y se centró en el relevamiento de una serie de variables como son el color, la textura de la superficie y la uniformidad del quemado. Los resultados obtenidos dispararon diversos interrogantes respecto a la idoneidad de esta metodología a la hora de evaluar prácticas de consumo, así como su complementariedad con otras variables consideradas, como pueden ser los patrones de fractura.

Palabras clave: Termoalteración, Cocción, Cazadores recolectores, Patagonia argentina 


\title{
Coloración ósea diferencial: el conjunto zooarqueológico del sitio La Mesada, Meseta Central de Santa Cruz
}

\author{
C. Valiza Davis ${ }^{1,2}$ y C. Bottari ${ }^{1}$ \\ ${ }^{1}$ División Arqueología, Facultad de Ciencias Naturales y Museo, Universidad Nacional de La Plata, Argentina. \\ catavaliza@hotmail.com; camibottari96@gmail.com \\ ${ }^{2}$ CONICET
}

Al analizar el conjunto óseo del sitio La Mesada (Localidad arqueológica La María) correspondiente al Holoceno Medio ( $c$ a. 4.500 AP), observamos que muchos de los restos presentaban significativamente, distintas coloraciones en su superficie.

Ante esta situación, discriminamos los materiales en una escala de colores, desde el marrón claro, pasando por el oscuro, hasta el negro. A partir de ello, confeccionamos un anexo informativo para el análisis de la coloración del conjunto, basándonos en las propuestas de Shahack-Gross y colaboradores (1997), Marin Arroyo y colaboradores (2008), López Gonzales y otros (2006). De esta manera, fueron aplicados, además del dato del color, los siguientes criterios de evaluación: la forma de expresión (mancha/moteado o cobertura); área afectada (en porcentaje); superficie afectada (cara externa, interna), intensidad de la coloración (uniforme o irregular) y por último la apariencia (brillo, mate o patina).

Durante este recorrido descriptivo, los materiales óseos con predominancia del color negro, fueron los que más preguntas nos ocasionaron. En un principio a estos elementos lo clasificamos como consecuencia del quemado, pero luego consideramos que estos podrían ser el resultado de otros procesos tafonómicos, como la tinción mineral producto del óxido de manganeso. Este interrogante deriva, por un lado, en que no pudimos observar macroscópicamente atributos propios de la termoalteracion. Por otro lado, esta incógnita cobra relevancia si consideramos el efecto de la acción del agua presente en el sitio. La presencia de éste también se evidenció, entre otros indicadores de formación de sitio, en un estudio funcional sobre artefactos líticos, donde no se registraron microrrastros de uso, dado que las superficies estaban alteradas por un lustre propio del accionar del agua.

De acuerdo a ello, en esta oportunidad queremos compartir con los asistentes del taller los materiales, las dudas e incógnitas, y el apéndice de análisis que hemos realizado, para poder avanzar en esta determinación concreta y conocer la historia tafonómica del conjunto, así como aportar a los conocimientos sobre los procesos de formación del sitio.

Palabras clave: Coloración superficial, Óxido de manganeso, Termoalteración, Apéndice de identificación 


\title{
Insectos en botella: hallazgo de restos en una letrina del siglo XIX
}

\author{
A. Igareta ${ }^{1,2,3}$, R. Mariani ${ }^{4}$, G. Varela ${ }^{4}$ y T. Teileche ${ }^{4}$ \\ ${ }^{1}$ División Arqueología, Facultad de Ciencias Naturales y Museo, Universidad Nacional de La Plata, Argentina. \\ ${ }^{2} \mathrm{HiTePAC}$, Facultad de Arquitectura y Urbanismo, Universidad Nacional de La Plata, Argentina. aigareta@gmail.com \\ ${ }^{3} \mathrm{CONICET}$ \\ ${ }^{4}$ División Entomología, Facultad de Ciencias Naturales y Museo, Universidad Nacional de La Plata, Argentina. \\ gracielavarela02@gmail.com; thel.teileche@fcnym.unlp.edu.ar
}

Durante los trabajos de excavación de los restos de una letrina utilizada en el siglo XIX ubicada en el sitio Moreno 550 en la ciudad de Buenos Aires, a 6 metros por debajo de la superficie actual del terreno, se hallaron y recuperaron docenas de botellas de vidrio enteras y perfectamente conservadas de vino, cerveza y ginebra, aguas carbonatadas, amargos y licores. En el interior de una de éstas se registró la presencia de cientos de pupas y puparios de moscas cuyo análisis reveló que se trataba de Musca domestica (Linnaeus), Muscina stabulans (Fallén), Megaselia scalaris (Loew) y Dohrniphora sp., especies saprófagas y sarcosaprófagas habitualmente utilizadas para la interpretación de contextos forenses y arqueológicos. Este hallazgo resulta una novedad para la arqueología histórica de la región, dado que la conservación de restos orgánicos en la misma resulta siempre compleja debido al elevado coeficiente de acidez y humedad de sus suelos. La preservación de los restos en el interior de una botella de vidrio obliga a considerar por qué el contenido de esa botella en particular atrajo a las moscas y posibilitó el desarrollo de su ciclo de vida, y que factores favorecieron luego su preservación en una unidad de descarte. Las especies registradas permiten inferir la presencia de materia orgánica en descomposición dentro de la botella antes de que fuera descartada. Por ser eusinantrópicas y por la estacionalidad que demuestran en la región, la colonización habría ocurrido durante un período cálido y, según los ciclos de vida que poseen, el sustrato habría permanecido descubierto entre 15 y 20 días. La presencia de pupas cerradas indica cambios en las condiciones ambientales que impidieron a algunos individuos completar su ciclo de vida y emerger como adulto, tal vez a consecuencia de su ingreso al ambiente anoxido y de bajas temperaturas de los depósitos de la letrina.

Palabras clave: Arqueología histórica, Insectos, Sinantropía 